\title{
Estado nutricional dos beneficiários do Programa Bolsa Família no Brasil - uma revisão sistemática
}

\author{
Nutritional status of beneficiaries of the "Bolsa Família" Program \\ in Brazil - a systematic review
}

Miriam Regina Wolf ${ }^{1}$

Antonio de Azevedo Barros Filho ${ }^{1}$

${ }^{1}$ Departamento de Pediatria, Faculdade de Ciências Médicas, Universidade Estadual de Campinas. Cidade Universitária Zeferino Vaz, Barão Geraldo. 13.083-887 Campinas SP Brasil. nutricionistamiriamwolf@ gmail.com
Abstract Various programs to combat malnutrition have been implemented over the years in Brazil. Since 2001, it has been the responsibility of the Bolsa Familia Program (BFP). Despite the expansion of BFP, to date there has been little coverage charting the advances and challenges to be faced as well as the impacts on health and nutrition of the population. Although the monitoring of nutritional status is one of the conditions for continuing to receive the benefit, when searching for this data, there is great difficulty in obtaining them. The scope of this systematic review is to obtain information on the nutritional status of the beneficiaries of the PBF, to grasp the national reality with regard to this situation, and assess whether the program had an impact on the nutritional status of the population served. A search was conducted online via Medline, Bireme, SciELO, Lilacs, University Libraries System (ULS) and Google. The search includes original articles and theses from March 2002 through May 2012 and approximately 100 works were located. After excluding the title there were 23 articles, and after reading the abstracts, 13 articles remained. The studies reviewed suggest that the Program is not modifying the nutritional status of the beneficiaries.

Key words Bolsa Família, Nutritional situation, Nutritional assessment, Anthropometry, Food and nutrition security
Resumo Diversos programas de combate à desnutrição foram implementados ao longo dos anos no Brasil. A partir de 2001 cabe ao Programa Bolsa Família (PBF) esta responsabilidade. Apesar da expansão do PBF ainda são pouca as avaliações que aquilatam os avanços e os desafios a serem enfrentados, bem como os impactos nas condições de saúde e nutrição da população. Ainda que o acompanhamento do estado nutricional seja uma das condicionalidades para a manutenção do recebimento do benefício, quando se buscam estes dados, há uma grande dificuldade em obtê-los. $O$ objetivo desta revisão sistemática é obter informações do estado nutricional dos beneficiários do PBF, para conhecer a realidade nacional no que diz respeito a esta situação, bem como avaliar se o programa causou impacto sobre o perfil nutricional da população atendida. Foi realizada uma busca através da Medline, Bireme, Scielo, Lilacs, Sistema de Bibliotecas Universitárias (SBU) e ferramenta Google. A busca inclui artigos originais e teses de março 2002 até maio de 2012. Encontrou-se aproximadamente 100 trabalhos. Após a exclusão pelo título restaram 23 artigos e após a leitura dos resumos, ficaram 13. Os trabalhos revisados sugererem que o Programa não está modificando o estado nutricional dos beneficiários. Palavras-chave Bolsa Família, Estado nutricional, Avaliação nutricional, Antropometria, Segurança alimentar 


\section{Introdução}

O Programa Bolsa Alimentação, atual Programa Bolsa Família (PBF), foi criado em 2001. Trata-se de um Programa de Transferência Condicionada de Renda. Estes programas foram desenvolvidos na América Latina e em todo mundo com o apoio do Banco Mundial.

O PBF trouxe diversas implicações para a sociedade brasileira, no âmbito social, econômico e político. Como nutricionista, estou focada nos estudos que avaliaram o estado nutricional dos beneficiários. Pois o combate à fome e desnutrição é um dos objetivos do programa ${ }^{1}$.

O Bolsa Família do Brasil é o programa de transferência monetária de maior envergadura no mundo ${ }^{1}$. Levando-se em conta os valores investidos neste programa, torna-se imperioso avaliar sua eficiência e efetividade, situação que pode ser extrapolada a outros países.

O benefício é distribuído a mais de $13 \mathrm{mi}-$ lhões de famílias, em todo território nacional, com rendimento de até $\mathrm{R} \$ 160,00$ per capita. O valor do benefício recebido por família pode variar entre $\mathrm{R} \$ 32,00$ e $\mathrm{R} \$ 306,00$, dependendo do número de crianças, adolescentes e gestantes. $\mathrm{O}$ Cadastro Único é o documento que identifica e caracteriza as famílias para o recebimento do PBF. A permanência no programa está vinculada ao cumprimento de algumas condicionalidades ${ }^{1}$. Que visam à frequência e assiduidade das crianças nas escolas, com o intuito de melhorar o nível educacional da população; acompanhamento periódico do estado nutricional e de saúde das famílias e a participação dos beneficiários nas ações de educação nutricional.

A não conformidade com as condições do programa pode causar sanções levando à exclusão do sistema de transferência. Para o cumprimento das condicionalidades da educação é fornecido um incentivo econômico para o adolescente frequentar a escola, cujo principal objetivo é reduzir o trabalho infantil. Porém, em muitos casos o recebimento do benefício é inferior ao valor que o adolescente receberia com o trabalho. Se de um lado aumenta a possibilidade de acesso à renda e redução futura da pobreza, por outro reduz a renda per capita familiar, aumentando o risco nutricional e de saúde. Já no caso do cumprimento das condicionalidades de saúde, não existe nenhum benefício financeiro imediato ${ }^{2}$.

Considerando as normas estabelecidas pelo governo, as diferentes esferas deveriam estar articuladas no sentido de alimentar os dados para a devida comprovação no cumprimento das con- dicionalidades. O acompanhamento de saúde e do estado nutricional das famílias é realizado pelas unidades básicas de saúde em cada município e estes dados são monitorados pelo Sistema de Vigilância Alimentar e Nutricional (SISVAN), que os repassa para o Ministério da Saúde. Ou seja, os dados de uma unidade básica de saúde na Amazônia, assim como de qualquer outra parte do Brasil, devem chegar até Brasília, serem processados e cruzados com os dados do Bolsa Família para que se comprove o cumprimento das condicionalidades.

Grande parte desses dados não são registrados; muitos dos beneficiários não compareçam às unidades de saúde. Desta forma, o controle no cumprimento das condicionalidades fica prejudicado, bem como nos seus objetivos que são melhorar o estado nutricional, a saúde e a educação da população. Fica evidente que a avaliação da efetividade do programa se defronta com muitas dificuldades.

Dada a importância da melhoria do estado nutricional da população, sobretudo no que tange à desnutrição materno-infantil, a evolução do estado nutricional dos beneficiários é uma forma de avaliação direta da efetividade do programa.

Segundo Weiss ${ }^{3}$, os interesses em realizar avaliação de projetos e programas são diversos. No governo, esses estudos estão diretamente ligados à questão da efetividade e da eficiência, e com o desempenho da gestão pública ${ }^{4}$.

Considerando que um dos objetivos do PBF é melhorar o estado nutricional dos beneficiários, sua efetividade pode ser avaliada através da melhoria desta condição em sua população-alvo.

Este trabalho tem o objetivo de obter informações do estado nutricional dos beneficiários do PBF e avaliar se o Programa causou impacto sobre o perfil nutricional da população atendida.

\section{Método}

Este artigo fez uma revisão sistemática de trabalhos científicos cujos objetivos foram avaliar o estado nutricional dos beneficiários do Programa Bolsa Família.

Foi realizada uma busca através da Medline, Bireme, Scielo, Lilacs, Sistema de bibliotecas universitárias e ferramenta Google de trabalhos com as seguintes palavras-chave: Bolsa Família/avaliação nutricional, Bolsa Família/antropometria, Bolsa Família/avaliação, Bolsa Família/segurança alimentar e nutricional, nos idiomas português, inglês e espanhol. 
A maioria dos artigos foi descartada pelo título, pois não se enquadrava no desejado.

Portanto, todos os trabalhos encontrados que realizaram avaliação nutricional dos beneficiários foram selecionados; independente do ano de publicação, autores ou tipo de estudo.

Restaram treze trabalhos que realizaram avaliação do estado nutricional dos beneficiários.

Critérios de inclusão: artigos que realizaram avaliação antropométrica dos beneficiários do PBF.

Critérios de exclusão: artigos com amostra muito pequena $(n=38)$, artigos que não realizaram ou utilizaram dados de avaliação antropométrica dos beneficiários.

\section{Resultados}

Foram encontrados doze estudos que avaliaram o estado nutricional dos beneficiários. Não foi encontrado nenhum estudo de coorte longitudinal que comparasse a evolução do estado nutricional dos beneficiários do programa, com os não beneficiários, ao longo do tempo. O Quadro 1 apresenta o resumo dos trabalhos.

\section{Discussão}

A avaliação e o acompanhamento do estado nutricional é uma das condicionalidades da perma-

Quadro 1. Resumo dos trabalhos pesquisados quanto ao estado nutricional dos beneficiários do Programa Bolsa Família.

\begin{tabular}{|c|c|c|c|c|c|}
\hline \multicolumn{6}{|c|}{ Estudos descritivos transversais } \\
\hline Estudos & Método & $\begin{array}{l}\text { Tipo de } \\
\text { estudo }\end{array}$ & Objetivos & $\operatorname{Amostra}(\mathrm{N})$ & Resultados \\
\hline $\begin{array}{l}\text { Estado nutricional de } \\
\text { população adulta } \\
\text { beneficiária do } \\
\text { Programa Bolsa } \\
\text { Família no município } \\
\text { de Curitiba, } \text { PR }^{5}\end{array}$ & $\begin{array}{l}\text { Aferido peso, } \\
\text { altura, } \\
\text { circunferência } \\
\text { da cintura e } \\
\text { cálculo IMC }\end{array}$ & $\begin{array}{l}\text { Descritivo } \\
\text { transversal } \\
\text { Julho } 2006 \\
\text { a julho } \\
2007\end{array}$ & $\begin{array}{l}\text { Descrição do } \\
\text { estado } \\
\text { nutricional da } \\
\text { população } \\
\text { adulta } \\
\text { beneficiária } \\
\text { PBF em } \\
\text { Curitiba- PR }\end{array}$ & $\begin{array}{l}747 \text { adultos a } \\
\text { partir de } 18 \\
\text { anos - ambos } \\
\text { os sexos }\end{array}$ & $\begin{array}{l}40 \% \text { eutróficos } \\
27,1 \% \text { obesos } \\
\text { segundo IMC }\end{array}$ \\
\hline $\begin{array}{l}\text { Programa bolsa } \\
\text { família: O } \\
\text { acompanhamento do } \\
\text { estado nutricional das } \\
\text { famílias titulares de } \\
\text { direito como forma } \\
\text { de enfrentar as } \\
\text { desigualdades sociais } \\
\text { no setor saúde }\end{array}$ & $\begin{array}{l}\text { Peso e } \\
\text { estaturadados } \\
\text { secundários } \\
\text { do SISVAN- } \\
\text { WEB }\end{array}$ & $\begin{array}{l}\text { Estudo } \\
\text { descritivo } \\
\text { transversal }\end{array}$ & $\begin{array}{l}\text { Prevalência de } \\
\text { desnutrição } \\
\text { infantil nos } \\
\text { beneficiários } \\
\text { do PBF nas } \\
\text { diferentes } \\
\text { regiões do } \\
\text { país, no } \\
\text { segundo } \\
\text { semestre de } \\
2006\end{array}$ & $\begin{array}{l}1.501 .537 \\
\text { crianças } \\
\text { menores de } \\
\text { sete anos em } \\
\text { todo o Brasil }\end{array}$ & $\begin{array}{l}\text { - Muito baixo peso } \\
\text { para idade }=2,12 \% \\
\text { - Baixo peso }= \\
6,74 \% \\
\text { - Risco sobrepeso = } \\
8,92 \% \text { no sudeste e } \\
5,11 \% \text { na região } \\
\text { norte } \\
\text { - Baixa estatura - } \\
16,8 \%\end{array}$ \\
\hline $\begin{array}{l}\text { Avaliação nutricional } \\
\text { de crianças } \\
\text { beneficiadas pelo } \\
\text { programa bolsa } \\
\text { família que } \\
\text { frequentam creches } \\
\text { municipais em Vitória } \\
\text { da Conquista, } \mathrm{BA}^{7}\end{array}$ & $\begin{array}{l}\text { Aferição de } \\
\text { peso e } \\
\text { estatura }\end{array}$ & $\begin{array}{l}\text { Descritivo } \\
\text { transversal }\end{array}$ & $\begin{array}{l}\text { Avaliação do } \\
\text { estado } \\
\text { nutricional } \\
\text { das crianças } \\
\text { beneficiadas } \\
\text { creches } \\
\text { municipais de } \\
\text { Vitória da } \\
\text { Conquista, BA }\end{array}$ & 228 & $\begin{array}{l}8,33 \% \text { obesas } \\
3,5 \% \text { sobrepeso } \\
66 \% \text { eutróficas } \\
21,49 \% \text { desnutrição } \\
\text { pregressa } \\
9,64 \% \text { desnutrição } \\
\text { Aguda }\end{array}$ \\
\hline
\end{tabular}


Quadro 1. continuação

\begin{tabular}{|c|c|c|c|c|}
\hline \multicolumn{5}{|c|}{ Estudos de coorte transversal } \\
\hline Estudos & Método & Objetivos & Amostra (N) & Resultados \\
\hline $\begin{array}{l}\text { Avaliação das condições } \\
\text { nutricionais de crianças } \\
\text { com base nos dados do } \\
\text { SISVAN Web e do } \\
\text { benefício do Programa } \\
\text { Bolsa Família no município } \\
\text { de Itajaí, SC }\end{array}$ & $\begin{array}{l}\text { Dados } \\
\text { secundários } \\
\text { SISVAN- } \\
\text { WEB }\end{array}$ & $\begin{array}{l}\text { Avaliação das } \\
\text { condições } \\
\text { nutricionais de } \\
\text { crianças com base } \\
\text { nos dados do } \\
\text { SISVAN WEB e } \\
\text { do benefício }\end{array}$ & $\begin{array}{l}3.327 \\
\text { crianças }\end{array}$ & $\begin{array}{l}\text { Diferenças expressivas no } \\
\text { índice } \mathrm{A} / \mathrm{I} \text {, sendo que os } \\
\text { beneficiários do PBF, } \\
\text { apresentaram } 12,3 \% \text { de } \\
\text { inadequação, contra } 7,4 \% \\
\text { dos não beneficiários }\end{array}$ \\
\hline $\begin{array}{l}\text { Alimentação, nutrição e } \\
\text { saúde em programas de } \\
\text { transferência de renda: } \\
\text { evidências para o Programa } \\
\text { Bolsa Família }{ }^{9}\end{array}$ & $\begin{array}{l}\text { Dados } \\
\text { secundários } \\
\text { de peso e } \\
\text { estatura, } \\
\text { convertidos } \\
\text { em P/I; } \\
\mathrm{P} / \mathrm{E} ; \mathrm{E} / \mathrm{I}\end{array}$ & $\begin{array}{l}\text { Avaliação do } \\
\text { estado nutricional, } \\
\text { insegurança } \\
\text { alimentar, } \\
\text { mortalidade } \\
\text { infantil }\end{array}$ & $\begin{array}{l}2.542 \\
\text { crianças }\end{array}$ & $\begin{array}{l}\text { Beneficiários - P/I = 47\% } \\
\text { adequados } \mathrm{P} / \mathrm{E}= \\
50,3 \% \% \text {; adequados } \\
\text { Não beneficiários - P/I = } \\
40 \% \text { e } \mathrm{P} / \mathrm{E}=46 \% \\
\mathrm{O} \text { resultado para a } \\
\text { amostra não pareada não } \\
\text { são significantes }\end{array}$ \\
\hline $\begin{array}{l}\text { Effects of a conditional cash } \\
\text { transfer programme on } \\
\text { child nutrition in Brazil }{ }^{10}\end{array}$ & $\begin{array}{l}\text { Aferição de } \\
\text { peso } \\
\text { estatura. } \\
\text { (Chamada } \\
\text { nutricional) }\end{array}$ & $\begin{array}{l}\text { Avaliar a relação } \\
\text { entre } \mathrm{PBF} \text { e o } \\
\text { estado nutricional } \\
\text { infantil }\end{array}$ & $\begin{array}{l}22.375 \\
\text { crianças de } \\
419 \\
\text { municípios } \\
\text { do norte e } \\
\text { nordeste }\end{array}$ & $\begin{array}{l}\text { Beneficiários tiveram } \\
26 \% \text { de maior } \\
\text { probabilidade de } \\
\text { apresentar peso e estatura } \\
\text { normal que os não } \\
\text { beneficiários }\end{array}$ \\
\hline $\begin{array}{l}\text { Estado nutricional e fatores } \\
\text { determinantes do déficit de } \\
\text { estatura em crianças } \\
\text { cadastradas no Programa } \\
\text { Bolsa Família. Município } \\
\text { Paula Cândido, } \mathrm{MG}^{11}\end{array}$ & $\begin{array}{l}\text { Peso e } \\
\text { estatura, } \\
\text { convertidos } \\
\text { em P/I; P/E; } \\
\text { E/IIMC }\end{array}$ & $\begin{array}{l}\text { Avaliar anemia e } \\
\text { distúrbio } \\
\text { antropométrico } \\
\text { entre beneficiários } \\
\mathrm{N}=263 \text { e não } \\
\text { beneficiários } \\
\mathrm{N}=184 \text { do } \mathrm{PBF}\end{array}$ & $\begin{array}{l}446 \text { crianças } \\
\text { de } 6 \text { a } 84 \\
\text { meses. } \\
\text { Divididas em } \\
\text { beneficiárias } \\
\text { e não } \\
\text { beneficiárias }\end{array}$ & $\begin{array}{l}\text { Prevalência de défcit } \\
\text { nutricional } \\
\text { Beneficiários - } \\
\mathrm{P} / \mathrm{I}=2,3 \% \\
\mathrm{P} / \mathrm{E}=0 \% \\
\mathrm{E} / \mathrm{I}=6,5 \% \\
\mathrm{IMC}=0,4 \% \\
\text { Não Beneficiários - } \\
\mathrm{P} / \mathrm{I}=1,6 \% \\
\mathrm{P} / \mathrm{E}=2,2 \% \\
\mathrm{E} / \mathrm{I}=6 \% \\
\mathrm{IMC}=0,5 \% \\
\text { Sem diferença estatística } \\
\text { entre os grupos }\end{array}$ \\
\hline $\begin{array}{l}\text { Avaliação antropométrica } \\
\text { e consumo alimentar em } \\
\text { crianças menores de cinco } \\
\text { anos residentes em um } \\
\text { município da região do } \\
\text { semiárido nordestino com } \\
\text { cobertura parcial do } \\
\text { Programa Bolsa Família }^{12}\end{array}$ & $\begin{array}{l}\text { Aferição } \\
\text { de peso e } \\
\text { estatura }\end{array}$ & $\begin{array}{l}\text { Avaliação do } \\
\text { estado nutricional } \\
\text { de crianças do } \\
\text { Semiárido } \\
\text { nordestino }\end{array}$ & $\begin{array}{l}198 \text { crianças } \\
\text { menores de } \\
\text { cinco anos }\end{array}$ & $\begin{array}{l}\text { Déficit de peso de } 4,3 \% \text { e } \\
\text { de altura } 9,9 \% \text { e excesso } \\
\text { de peso em } 14,0 \% \text {. } \\
\text { Sem diferenças estatísticas } \\
\text { entre estado nutricional } \\
\text { de beneficiárias e não } \\
\text { beneficiárias. Em ambos } \\
\text { os grupos, os consumos } \\
\text { de frutas, verduras e } \\
\text { legumes foram baixos e } \\
\text { semelhantes entre si. As } \\
\text { crianças do programa } \\
\text { bolsa família têm risco } \\
\text { três vezes maior de } \\
\text { consumir guloseimas }\end{array}$ \\
\hline
\end{tabular}




\begin{tabular}{|c|c|c|c|c|c|c|}
\hline \multicolumn{7}{|l|}{ Quadro 1. continuação } \\
\hline \multicolumn{7}{|c|}{ Estudos longitudinais } \\
\hline Estudos & Método & Objetivos & Amostra $(\mathrm{N})$ & \multicolumn{3}{|c|}{ Resultados } \\
\hline $\begin{array}{l}\text { Perfil do estado } \\
\text { nutricional de crianças } \\
\text { de zero a sete anos } \\
\text { beneficiárias do } \\
\text { programa bolsa família } \\
\text { no período de } 2008 \text { e } \\
2009 \text { no município de } \\
\text { Paulista-PE }\end{array}$ & $\begin{array}{l}\text { Dados } \\
\text { secundários } \\
\text { do SISVAN- } \\
\text { WEB }\end{array}$ & $\begin{array}{l}\text { Descrição do } \\
\text { estado nutricional } \\
\text { de crianças } \\
\text { beneficiárias o } \\
\text { PBF em Paulista, } \\
\text { PE }\end{array}$ & $\begin{array}{l}6.700 \\
\text { crianças }\end{array}$ & \multicolumn{3}{|c|}{$\begin{array}{l}\mathbf{2 0 0 8} \\
\text { Baixo peso - 3,15\% } \\
\text { Peso elevado - 9,57\% } \\
\text { Baixa estatura - 6,37\% } \\
\text { Obesidade - 5,8\% } \\
\mathbf{2 0 0 9} \\
\text { Baixo peso - 3,43\% } \\
\text { Peso elevado - 10,97\% } \\
\text { Baixa estatura - 6,74\% } \\
\text { Obesidade - } 6,7 \%\end{array}$} \\
\hline $\begin{array}{l}\text { Programas de } \\
\text { transferência } \\
\text { condicionada de renda } \\
\text { e seu impacto sobre o } \\
\text { estado nutricional de } \\
\text { crianças e adultos na } \\
\text { região nordeste do } \\
\text { Brasil }^{14}\end{array}$ & $\begin{array}{l}\text { Dados } \\
\text { secundários } \\
\text { da Pesquisa } \\
\text { de } \\
\text { orçamento } \\
\text { familiar } \\
\text { POF- 2002/ } \\
2003\end{array}$ & $\begin{array}{l}\text { Avaliação do } \\
\text { impacto PBF } \\
\text { sobre o estado } \\
\text { nutricional de } \\
\text { crianças e adultos } \\
\text { do nordeste } \\
\text { brasileiro }\end{array}$ & $\begin{array}{l}5.267 \\
\text { crianças } \\
18.806 \\
\text { adultos }\end{array}$ & \multicolumn{3}{|c|}{$\begin{array}{l}\text { Crianças baixo peso }=4,6 \% \\
\text { Adolescentes - excesso de peso } \\
=16.7 \% \text { e baixo peso }=3 ; 7 \% \\
\text { Conclusão: Valores baixos e } \\
\text { insuficientes para produzirem } \\
\text { o impacto esperado } \\
\text { principalmente entre adultos e } \\
\text { crianças mais velhas e aquelas } \\
\text { vivendo em zona rural }\end{array}$} \\
\hline $\begin{array}{l}\text { Perfil nutricional dos } \\
\text { beneficiários do PBF } \\
\text { Tabatinga, AM } \\
2006 \text { e } 2008^{15}\end{array}$ & $\begin{array}{l}\text { Dados } \\
\text { secundários } \\
\text { peso e } \\
\text { estatura }\end{array}$ & $\begin{array}{l}\text { Avaliação } \\
\text { nutricional dos } \\
\text { beneficiários } \\
\text { (adultos e } \\
\text { crianças) }\end{array}$ & $\begin{array}{l}\mathrm{N}=636 \\
\text { em } 2006 \\
\mathrm{~N}=1.885 \\
\text { em } 2008\end{array}$ & \multicolumn{3}{|c|}{$\begin{array}{l}\mathbf{2 0 0 6} \\
22 \% \text { de desn. crônica } \\
235 \text { risco nut.; } \\
53 \% \text { eutróficos } \\
\mathbf{2 0 0 8} \\
32 \% ; 20 \% \text { e } 44 \% \\
\text { respectivamente }\end{array}$} \\
\hline $\begin{array}{l}\text { Análise do impacto do } \\
\text { programa bolsa família } \\
\text { em relação ao estado } \\
\text { nutricional de crianças } \\
\text { de zero a sete anos } \\
\text { pertencentes às } \\
\text { famílias beneficiárias } \\
\text { do programa no } \\
\text { município de Pará de } \\
\text { Minas, MG, no } \\
\text { período de } 2007 / \\
2008^{16}\end{array}$ & $\begin{array}{l}\text { Levantamento } \\
\text { retrospectivo } \\
\text { de dados } \\
\text { secundários. }\end{array}$ & $\begin{array}{l}\text { Descrição do } \\
\text { estado nutricional } \\
\text { de crianças } \\
\text { beneficiárias o } \\
\text { PBF em Pará de } \\
\text { Minas, MG }\end{array}$ & $\begin{array}{l}563 \text { crianças } \\
\text { de zero a sete } \\
\text { anos }\end{array}$ & \multicolumn{3}{|c|}{$\begin{array}{l}2007 \\
\text { Baixo peso - 8,6\% } \\
\text { Risco nut. - 7,\% } \\
\text { Eutrófico - 77,9\% } \\
\text { Sobrepeso - 6,3\% } \\
\mathbf{2 0 0 8} \\
\text { Baixo peso - 5,5\% } \\
\text { Risco nut. - 8,7\% } \\
\text { Eutrófico - 77,3\% } \\
\text { Sobrepeso - 8,41\% } \\
\text { Redução da desnutrição e } \\
\text { aumento sobrepeso }\end{array}$} \\
\hline $\begin{array}{l}\text { O Programa Bolsa } \\
\text { Família na rede } \\
\text { municipal de saúde } \\
\text { Uberlândia }^{17} \\
2006 \text { e } 2007\end{array}$ & $\begin{array}{l}\text { Dados } \\
\text { secundários } \\
\text { SISVAN- } \\
\text { WEB }\end{array}$ & $\begin{array}{l}\text { Descrição do } \\
\text { estado nutricional } \\
\text { de famílias } \\
\text { beneficiárias o } \\
\text { PBF em } \\
\text { Uberlândia, MG }\end{array}$ & $\begin{array}{l}3.860 \\
\text { crianças }\end{array}$ & $\begin{array}{l}\text { M. baix P. } \\
\text { Baixo peso } \\
\text { Risc. nut. } \\
\text { Eutrófico } \\
\text { Sobrepeso }\end{array}$ & $\begin{array}{r}2006 \\
1,9 \% \\
3,93 \% \\
8,27 \% \\
77,6 \% \\
8,88 \%\end{array}$ & $\begin{array}{c}2007 \\
1,01 \% \\
4,17 \% \\
8,03 \% \\
79,02 \% \\
7,17 \%\end{array}$ \\
\hline
\end{tabular}

nência dos beneficiários no programa, pois um dos objetivos do PBF é reduzir a fome no Bra$\operatorname{sil}^{18,19}$. A fome, a falta de alimentação em quanti- dade e qualidade adequadas leva à desnutrição ${ }^{20}$, portanto, a avaliação do estado nutricional ao longo do tempo de permanência do beneficiário 
no programa é uma forma direta de mensurar a sua efetividade. Considerando que:

- O acompanhamento do estado nutricional deveria ser realizado sob pena de exclusão do benefício;

- Estes dados deveriam ser monitorados pelo governo no sentido de se fazer cumprir as condicionalidades;

- Entendendo que o governo é o responsável pelo repasse de verbas públicas e, em última análise, o principal interessado em atingir as metas propostas e demonstrá-las à sociedade.

Os dados disponíveis no site oficial do PBF encontram-se muito desatualizados. Portanto, esta avaliação depende de liberação do acesso aos dados do SISVAN de cada município. Porém grande parte dos municípios não possui este sistema implantado e/ou atualizado. Considerando estas dificuldades fica claro o motivo de tão poucos trabalhos publicados.

Os resultados dos programas de transferência dependem crucialmente do cumprimento pelos beneficiários das condicionalidades e da garantia do acesso aos seus sistemas. Estas condições deveriam evitar que as transferências monetárias sejam usadas de forma inadequada e, portanto, apresentem resultados insatisfatórios quanto ao estado de saúde e educação dos beneficiários.

A adesão às condicionalidades é altamente dependente do acesso aos serviços. A imposição de condições não é eficaz se não for acompanhada por investimentos para garantir a prestação dos serviços que atendam a demanda. A qualidade e a disponibilidade dos serviços de saúde são a chave para alcançar as metas de saúde de qualquer programa de transferência condicional monetária. Portanto, a eficácia de programas como o PBF é diretamente proporcional à qualidade dos serviços disponiveis. Quando estas condições não existem, uma transferência de renda não gerará quaisquer resultados em termos de saúde/nutrição.

Essas considerações são particularmente relevantes para algumas regiões do Brasil.

Apesar de que quase $70 \%$ das famílias reporta incremento da variedade de alimentos consumidos, cabe destacar o aumento no consumo de alimentos altamente calóricos e de baixo valor nutritivo afeta especialmente as famílias com estado de insegurança alimentar grave. Particularmente, o incremento do consumo destes alimentos é proporcionalmente maior que do de frutas e verduras ${ }^{2}$. Desta forma, a redução da desnutrição tem sido acompanhada do aumento de sobrepeso e da anemia, refletindo consumo inadequado de alimentos.

Este dado reflete a necessidade da implementação sistemática das ações de educação alimentar em todas as unidades básicas de saúde, sob a supervisão de um profissional nutricionista conforme determinam o Conselho Federal de Nutricionistas e as condicionalidades do PBF.

A avaliação mais fácil, rápida e direta seria buscar o peso e estatura das crianças. Considerando que estas devam ser acompanhadas pelas unidades de saúde para continuar recebendo o benefício, estes dados deveriam estar facilmente disponíveis, pois os de consumo alimentar são por sua natureza de mais difícil comprovação.

Limitações o estudo: Tratando-se de avaliação antropométrica, sempre existe a possibilidade de erro humano por falta de treinamento e de acompanhamento e metodologia adequados. As avaliações encontradas não fazem o acompanhamento do desenvolvimento do estado nutricional da população-alvo, ao longo do tempo, comparando com os não beneficiários (coorte longitudinal). Portanto, fica difícil avaliar o impacto do programa sobre a antropometria dos beneficiários.

\section{Conclusão}

Os trabalhos revisados parecem sugerir que o efeito do PBF sobre o estado nutricional dos beneficiários não está de acordo com o esperado pelos objetivos do programa.

O levantamento bibliográfico realizado aponta a dificuldade em encontrar dados que demonstrem a eficiência e a eficácia do PBF. Mesmo nos municípios onde o Sistema de Vigilância Alimentar e Nutricional (SISVAN) encontra-se informatizado com ligação direta com o SISVAN nacional, o cruzamento de dados com o PBF não ocorre. A dificuldade na obtenção de dados para a avaliação do estado nutricional, ao longo do tempo em que o indivíduo se beneficia ou beneficiou do PBF, é o maior obstáculo para as pesquisas. Neste sentido fica o desafio para a realização de trabalhos científicos que possam de fato avaliar a evolução do estado nutricional dos beneficiários, ressaltando que a avaliação deste é apenas uma das possíveis a serem implementadas para o PBF. 


\section{Colaboradores}

MR Wolf e AAB Barros Filho participaram igualmente de todas as etapas de elaboração do artigo.

\section{Referências}

1. Brasil. Ministério do Desenvolvimento Social e Combate à Fome (MDS). Secretaria Nacional de Renda de Cidadania (Senarc). Programa Bolsa família. [sd] [acessado 2010 out 28]. Disponível em: http://www.mds.gov.br/bolsafamilia.

2. Castiñeira BR, Nunes LC, Rungo P. Impacto de los programas de transferencia condicionada de renta sobre el estado de salud: el Programa Bolsa Familia de Brasil. Rev Esp Salud Publica 2009; 83(1):85-97.

3. Weiss CH . Theory-based evaluation: Past, present, and future. Hoboken: Wiley Periodicals, Inc; 2004.

4. Costa NR. A proteção social no Brasil: universalismo e focalização nos governos FHC e Lula. Cien Saude Colet 2009; 14(3):693-706.

5. Lima FEL, Rabito EI, Dias MRMG. Estado nutricional de população adulta beneficiária do Programa Bolsa Família no município de Curitiba, PR. Rev Bras Epidemiol 2011; 14(2):198-206.

6. Duar H, Toral N, Coutinho J. Programa Bolsa Família: o acompanhamento do estado nutricional das famílias titulares de direito como forma de enfrentar as desigualdades sociais no setor saúde. III Jornada Internacional de Políticas Públicas; 2007 ago 28-30. São Luís: Universidade Federal do Maranhão; 2007.

7. Mendes BAB, Kluge J, Amaral KM, Silva PS. Avaliação nutricional de crianças beneficiadas pelo programa bolsa família que frequentam creches municipais em Vitória da Conquista- BA. Biblioteca Virtual Bolsa Família, 2008. [acessado 2012 abr 5]. Disponível em: http://www.ipc-undp.org/mds.do.

8. Monestel A. Avaliação das condições nutricionais de crianças com base nos dados do SISVAN Web e do benefício do Programa Bolsa Família no município de Itajaí-SC. Itajaí: Univali; 2011.

9. Camelo RS, Tavares PA, Saiani CCS. Alimentação, Nutrição e Saúde em Programas de Transferência de Renda: Evidências para o Programa Bolsa Família. 2006. [Internet]. [acessado 2012 jun 23]. Disponível em: http://www.anpec.org.br/revista/vol10/ vol10n4p685_713.pdf.

10. Paes-Sousa R, Santos LMP, Miazaki ES. Effects of a conditional cash transfer programme on child nutrition in Brazil. Bull World Health Organ 2011; 89(7):496-503.

11. Oliveira FCC, Cotta RMM, Ribeiro AQ, Sant'Ana LFR, Priore SE, Franceschini SCC. Estado nutricional e fatores determinantes do déficit estatural em crianças cadastradas no Programa Bolsa Família. Epidemiol Serv Saúde 2011; 20(1):7-18.

12. Saldiva SRDM, Silva LFF, Saldiva PHN. Avaliação antropométrica e consumo alimentar em crianças menores de cinco anos residentes em um município da região do semiárido nordestino com cobertura parcial do programa bolsa família. Rev Nutr 2010; 23(2):221-229.

13. Aguiar NA. Perfil do estado nutricional de crianças de zero a sete anos beneficiárias do programa bolsa família no período de 2008 e 2009 no município de Paulista-PE [monografia]. Recife: Centro de Pesquisas Aggeu Magalhães; 2010. 
14. Souza ALM. Programas de transferência condicionada de renda e seu impacto sobre o estado nutricional de crianças e adultos na região nordeste do Brasil [tese]. São Paulo: Universidade de São Paulo; 2009.

15. Santillan R, Bazarelli A, Naupay R, Nagaham D. Implantação da Coordenação Municipal de Alimentação e Nutrição no Município de Tabatinga / AM. $2^{a}$ Mostra de experiências bem sucedidas em Nutrição. Tabatinga; 2009 [acessado 2012 jul 19] Disponível em: http://nutricao.saude.gov.br/evento/2mostra/mostra_trabalho_rel.php?cod $=5252$

16. Faria LAN, Pena APSA. Análise do impacto do programa bolsa família em relação ao estado nutricional de crianças de zero a sete anos pertencentes às famílias beneficiárias do programa no município de Pará de Minas/MG, no período de 2007/2008. SynThesis Revista Digital FAPAM. 2008;1(1):355-354. [acessado: 2012 jun 19]. Disponível em: www.fapam. edu.br/revista.

17. Lopes DD, Couto EP, Moreira TCSA. O Programa Bolsa Família na rede municipal de saúde: o caso do município de Uberlândia. XIII Seminário sobre a Economia Mineira; Uberlândia; 2008.

18. Costa E, Romani SAM, Batista Filho M, Rocha AN. Desnutrição recente, crônica e pregressa em quatro localidades do Estado de Pernambuco, Brasil. Rev Saude Publica 1981; 15(2):211-220.

19. Estrella J, Ribeiro LM. Qualidade da gestão das Condicionalidades do Programa Bolsa Família: uma discussão sobre o índice de gestão descentralizada. Rev Adm Pública 2008; 42(3):625-641.

20. Senna MCM, Burlandy L, Monnerat GL, Schottz V, Magalhães R. Programa bolsa família: nova institucional idade no campo da política social brasileira? Rev Katálysis 2007; 10(1):86-94.

Artigo apresentado em 15/03/2013

Aprovado em 22/04/2013

Versão final apresentada em 24/04/2013 